\title{
ASSOCIATION BETWEEN WEIGHT STATUS AND BODY IMAGE PERCEPTION AMONG FEMALE ADOLESCENTS IN TERENGGANU
}

\author{
RHEHANA MOHD AYOB ${ }^{1}$ and BEE SUAN WEE ${ }^{1}$ \\ ${ }^{1}$ School of Nutrition and Dietetics, Faculty of Health Sciences, University Sultan Zainal Abidin, \\ 21300 Kuala Nerus, Terengganu, Malaysia \\ *E-mail: beesuan@unisza.edu.my
}

Accepted 9 November 2021, Published online 30 November 2021

\begin{abstract}
Body image is the picture of how our body is perceived and stored in our minds. During puberty, many changes occur in the body of adolescents. Often, a change in physical appearance has an impact on the levels of the body's satisfaction. Thus, it is crucial to assess the body image among adolescents to ensure the wellbeing of both physical and mental health is at the optimal stage. Therefore, the present study aims to determine the body fat percentage of female adolescents. Then, to evaluate the association between weight status and body image perception. Lastly, to assess whether the attitudes towards body image are predictive of female adolescents' weight status. This cross-sectional study was conducted among 198 female adolescents in Kuala Nerus, Terengganu. Anthropometric measurements included weight and height, and BMI was determined. Body composition was assessed by using Bio-electrical Impedance Analysis through Bodystat Quadscan 4000 instrument. Body image perception was measured using Stunkard's Figure Rating Scale and Attitudes towards Body Image that was adapted from the Multidimensional Body Self Relation Questionnaire (MBSRQ). A total of $27.8 \%$ of female adolescents were overweight and $1.0 \%$ were obese by body fat. Among the female adolescents, $62.5 \%$ were dissatisfied with their body image, although $57.5 \%$ of them were in the normal weight category. Pearson's Chi-Square test revealed there is an association between weight status and body image perception among female adolescents $(p<0.045)$. Based on the Multiple Linear Regression Analysis, there is a significant linear relationship between attitudes towards body image, the domain of comparison of body shape, and BMI $(p<0.005)$. Thus, for every 1 unit increase in the domain of comparison of body shape, there will be a 0.570 decrease in BMI. Comparison of body shape is a significant predicting factor for BMI. In conclusion, further study should be carried out to investigate the factors that can lead to the development of dissatisfaction with body image and the effects of getting negative body image in life of the adolescents during their adolescent's phase.
\end{abstract}

Key words: Body fat percentage, BIA, body image perception, Stunkard's Figure Rating Scale

\section{INTRODUCTION}

The revolution of body image preferences among females can be seen in the era of the Italian Renaissance, a full body with light hair and skin is defined as the beauty standard that many women want to achieve. But then, moving to the 1920 s, where being thin and in the shape of the hourglass is believed to be the beauty standard. Moving forward to this era, women's preferences changed towards a flat tummy, healthy skinny, large breasts, and butt with thigh gap (Vannesa, 2014). All of this shows that women's preferences are constantly

* To whom correspondence should be addressed. changing and it is crucial to address these changes to ensure that adolescents grow mentally and physically healthy.

According to David (1994), "body image" refers to "the picture of our own body which we form in our mind, that is to say, how the body appears to ourselves". Negative body image is perceived as body image disturbance, body dissatisfaction, and overly occupied body image (Tylka \& WoodBarcalow, 2015). Body composition, if described from the chemical perspective, refers to the fat, protein, minerals, and body water contained in the body or if seen from tissue and organ perspectives, described as physical characteristics and function. In Chengdu, China a study conducted among 517 adolescents 
reported that normal and overweight adolescents reported greater body dissatisfaction than underweight adolescents (Xu et al., 2010). A study among male and female college students at Oklahoma State University, presented that body image development in both males and females was highly influenced by body composition (Crishel, 2006). A study done among Mexican-American female adolescents showed that females with higher body fat percentages possess greater body dissatisfaction (Guinn et al., 1997). Another study indicated that the weight status of adolescents is linked to body image as higher Body Mass Index (BMI) is associated with increased weight concerns among adolescents of both genders (Reel et al., 2015).

According to the National Health Morbidity Survey (NHMS) of 2019, the prevalence of overweight and obesity for the adult population was $30.4 \%$ and $19.7 \%$, respectively (IPH, 2019). Trends of overweight, obesity, and abdominal obesity have been raised from the year 2011 until 2019 as the number increased from $29.4 \%, 15.1 \%$, and $43.0 \%$ to $30.4 \%, 19.7 \%$, and $52.6 \%$, respectively (IPH, 2019; IPH, 2011). As for the children and adolescents aged between 5 to 17 years old, the prevalence of overweight and obese are $15.0 \%$ and $14.8 \%$, respectively (NIH, 2019) Based on WHO definition, adolescents are those aged from 10 to 19 years old (WHO, 2021) while the definition for children and preadolescents refers to those aged below 12 years old (Brown 2019). There was a lacking of research in the area of body image and body composition, especially among adolescents in the East Coastal area of Malaysia particularly in Terengganu as previous studies conducted focused on adolescents in Kedah and Pulau Pinang (Khor et al., 2009). Therefore, this study aimed to determine the body fat percentage of female adolescents. Then, to evaluate the association between weight status and body image perception. Lastly, to assess whether the attitudes towards body image are predictive of female adolescents' weight status. This cross-sectional study explored how body image perception could further affect the body composition among female adolescents in Kuala Nerus. So, the findings in this study can later be used to tackle the perception of body image among adolescents towards attaining healthy body composition and a positive body image.

\section{MATERIALS AND METHODS}

\section{Study setting}

This cross-sectional study was conducted in the primary and secondary schools located in the district of Kuala Nerus in Terengganu, Malaysia.

\section{Study sample}

The sampling method used in this study is probability sampling which is single-stage cluster sampling. There are 5 schools selected that are from those located in Kuala Nerus district. Then, random classes were chosen from primary year 4 to year 5 and secondary from form 4 and 5 to participate in this study. The sample size was calculated using the Epi info version 7 software using the Odds Ratio (OR), for overestimated and underestimated weight status were 14.47 and 5.92, respectively. The sample size calculated was 58 and 162 subjects according to the OR calculated. These OR were taken from a previous study conducted among African American adolescents (Wang \& Chen, 2011). After taking into consideration of the $20 \%$ drop-out rate, the proposed sample size was 203 participants. The final total sample size achieved was 198 female adolescents with an age range between 10 to 16 years old. The inclusion criteria were female adolescents, able to read and understand Malay and English languages. The participants must have obtained parental consent to participate in this study. Lastly, the participants must be healthy and free from any medical condition.

\section{Ethical consideration}

This study was approved by Universiti Sultan Zainal Abidin Ethics Committee with the referral code of UniSZA.C/2/UHREC/628-2(93). This study was also approved by the Ministry of Education (MOE) with the referral code of KPM.600-3/2/3eras(4527). In addition, this study also obtained approval from the Kuala Terengganu State Education Department with the referral code of P.T. 0603035(24). The details of the purpose and procedure of the study were explained to the respondents together with written and verbal consent obtained from them. Private information and confidentiality were ensured. The data collected was only accessible to the researcher.

\section{Procedures}

Based on the registry list provided by the school administration, all the participants were screened for eligibility to fulfill all the inclusion criteria. Participants that fulfilled all the inclusion criteria were briefed about the objectives and the protocol of this study. The participants were given a consent form and they were asked to bring the consent form home and ask for their parent's agreement to participate in this study. After obtaining the consent form from the students, the participants were asked to fill out the socio-demographic questionnaire to obtain background information of the study participants. The outlined study protocol was that 
the participants need to get enough sleep of 8 hours, be fasted, and also had to empty their bladder before the body composition measurements were taken.

During data collection, a series of measurements were done including personal information, anthropometric and body composition measurements. Participants were also asked to fill out body image perception using Stunkard's Figure Rating Scale and Attitudes toward body image questionnaire (Khor, 2009). The Stunkard's Figure Rating Scale was used to assess the body image perception of female adolescents about how they perceive their body image as the ideal body shape and current body shape. The Stunkard's Figure Rating Scale uses the body silhouette method to assess body image perception. It shows nine body silhouette figures of women, from very thin to very obese. The participants chose the figure which was closest to their own body along with the silhouette they would like to have. The results allowed three measurements. The current image, the desired image, and the discrepancy (desired-current), which was interpreted as the level of dissatisfaction with the body image. If the discrepancy was equal to 0 , the participants were satisfied with their body image; if the discrepancy had a positive value, the participants wished to be bigger; if the discrepancy had a negative value, the participants wished to be thinner. A positive and negative value would indicate that the participants are dissatisfied with their body image and a 0 value shows that the participants are satisfied with their body image. The Attitudes toward body image questionnaire was used to assess the factors affecting BMI and body fat percentage (Khor, 2009). The questionnaire consists of 28 items in total. The questionnaire comprises of 9 subscales or domains which are parental acceptance, peer acceptance, the importance of body shape, comparison of body shape, body shape confidence, body shape satisfaction, bodyweight satisfaction, body weight, and shape anxiety, and body weight and shape preoccupation. The questionnaire is based on the 5 points Likert scale which ranges from 1 to 5 , where 1 indicates disagree, while 5 indicates agree. It demonstrated good validity and reliability of body image perception evaluation especially among the population of Malaysia with the internal consistency of 0.77 and $\alpha$-Cronbach's value was 0.48-0.85 (Khor, 2009). As for the anthropometric measurement, the height $(\mathrm{cm})$, weight $(\mathrm{kg})$, waist $(\mathrm{cm})$, and hip measurement $(\mathrm{cm})$ of the participants were taken according to the standard study protocol. Height was measured without shoes to the nearest $0.1 \mathrm{~cm}$, weight was measured without shoes and with light clothing to the nearest $0.1 \mathrm{~kg}$ while waist circumference was measured to the nearest $0.1 \mathrm{~cm}$. Lastly, the body composition was assessed through
Bioelectrical Impedance Analysis (BIA) method for body fat percentage, fat mass, and fat-free mass. The cut-offs for normal body fat percentage for adolescents aged between 10 to 12 are $26 \%, 28 \%$, and $30 \%$ respectively. While the adolescents aged 13 to 15 years old are $33 \%, 34 \%$, and $34 \%$ respectively (Taylor et al., 2002). The cut-offs were obtained from the comparison conducted between the method of Dual-energy X-ray absorptiometry (DEXA) and BMI. BIA has four electrodes on each footplate and hand handle. It measures impedance at five segments of the body which are the whole body, both feet and hands by allowing the current and voltage to flow between hand and feet to quantify the percentage of body fat and muscle mass.

\section{Statistical analysis}

Data collected from this study were analyzed using the IBM SPSS Software version 20 (IBM Corp, New York) to obtain descriptive and inferential statistics. A normality test was conducted to recognize if the data were normally distributed. The alpha (á) level was set at $(p<0.05)$. If the $\mathrm{p}$-value is equal to or less than the á value, the null hypothesis $\left(\mathrm{H}_{\mathrm{o}}\right)$ will be rejected. The confidence level was set up at a $95 \%$ confidence interval. Descriptive, Pearson's Chi-Square test and Multiple Linear Regression were used to analyze the data obtained. Coefficient determination $\left(\mathrm{R}^{2}\right)$ was used to determine the goodness of fit for the regression analysis.

\section{RESULTS}

\section{Description of participants}

Among 198 participants, most of them were aged 15 to 16 years old which accounted for $42.4 \%$. The majority of the respondents were from lower-income families (B40) which is $62.6 \%(n=124)$. The median BMI was $21.3 \mathrm{~kg} / \mathrm{m}^{2}(6.5)$ and mean body fat $28.6 \%$ (7.3) of the respondents were in the normal category (Table 1).

\section{Body fat measurement using BIA}

The majority $(71.2 \%)$ of the participants had a normal range for body fat percentage which accounts for 141 participants and only $27.8 \%(n=55)$ were overweight and $1.0 \%(n=2)$ were obese by body fat percentage (Figure 1).

\section{Association between weight status and body image perception}

The weight status was categorized according to WHO growth reference (WHO 2007), as depicted in Figure 2. Despite being in the normal BMI category, the prevalence of respondents who feel dissatisfied with their body image is $57.5 \%$. For all the BMI 
Table 1. Socio-demographic characteristics and anthropometric measurements of the respondents $(n=198)[n$ (\%); mean (SD); median (IQR)]

\begin{tabular}{lc}
\hline Characteristics & $\begin{array}{c}\text { Female } n(\%) \\
\text { mean (SD); } \\
\text { median (IQR) }\end{array}$ \\
\hline Age group (years old) & \\
$10-11$ & $41(20.7)$ \\
$13-14$ & $73(36.9)$ \\
$15-16$ & $84(42.4)$ \\
Mean (SD) & $13.6(2.0)^{\mathrm{a}}$ \\
& \\
Family Income & \\
Low income (B40) (<RM4849) & $124(62.6)$ \\
Middle income (M40) (RM4850-RM10959) & $36(18.2)$ \\
High income (T20) (>RM10960) & $38(19.2)$ \\
& \\
Anthropometry & \\
Weight (kg) & $49.7(16.5)^{\mathrm{b}}$ \\
Height (m) & $1.5(11.3)^{\mathrm{b}}$ \\
BMl (kg/m $\left.{ }^{2}\right)$ & $21.3(6.5)^{\mathrm{b}}$ \\
Body fat (\%) & $28.6(7.3)^{\mathrm{a}}$ \\
Fat mass & $13.4(9.8)^{\mathrm{b}}$ \\
Fat-free mass & $33.7(8.3)^{\mathrm{b}}$ \\
\hline
\end{tabular}

a mean (SD).

${ }^{\mathrm{b}}$ median (IQR)

groups, the majority of them were dissatisfied with their body image. The body image perception was assessed using the 9-figure silhouette scale.

As for the adolescents who have normal BMI and are satisfied with their body image, is $73.0 \%$ meanwhile the adolescents who are also in normal BMI but dissatisfied with their body image, is $58.9 \%$
(Table 2). This means that the participants are more dissatisfied with their body image despite their BMI being in the normal category or not the normal category. There are significant associations between body image perception and weight status $(p=0.045)$.

\section{Association between 9 domains of attitudes towards body image}

Based on the 9 domains taken from the questionnaire, attitudes toward body image (parental acceptance, peer acceptance, importance of body shape, comparison of body shape, body shape confidence, body shape satisfaction, bodyweight satisfaction, body weight, and shape anxiety and body weight and shape preoccupation), only 1 domain of comparison of body shape has a

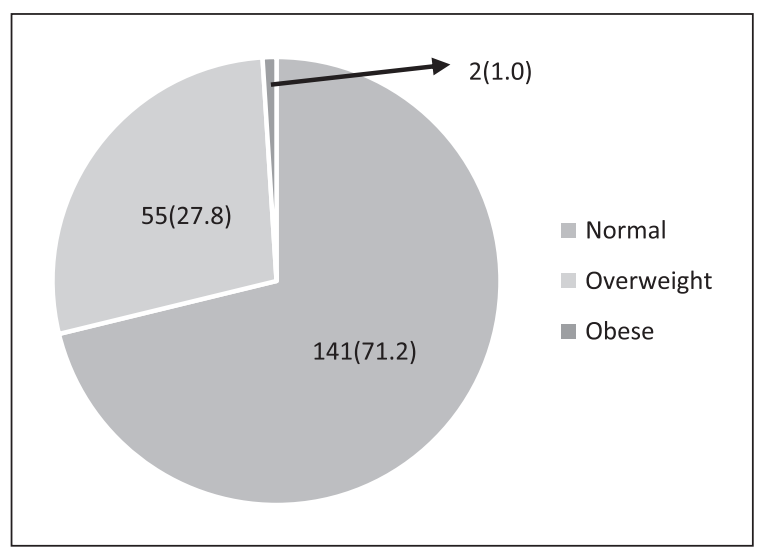

Fig. 1. Body Fat percentage of the female adolescents $n(\%)$.

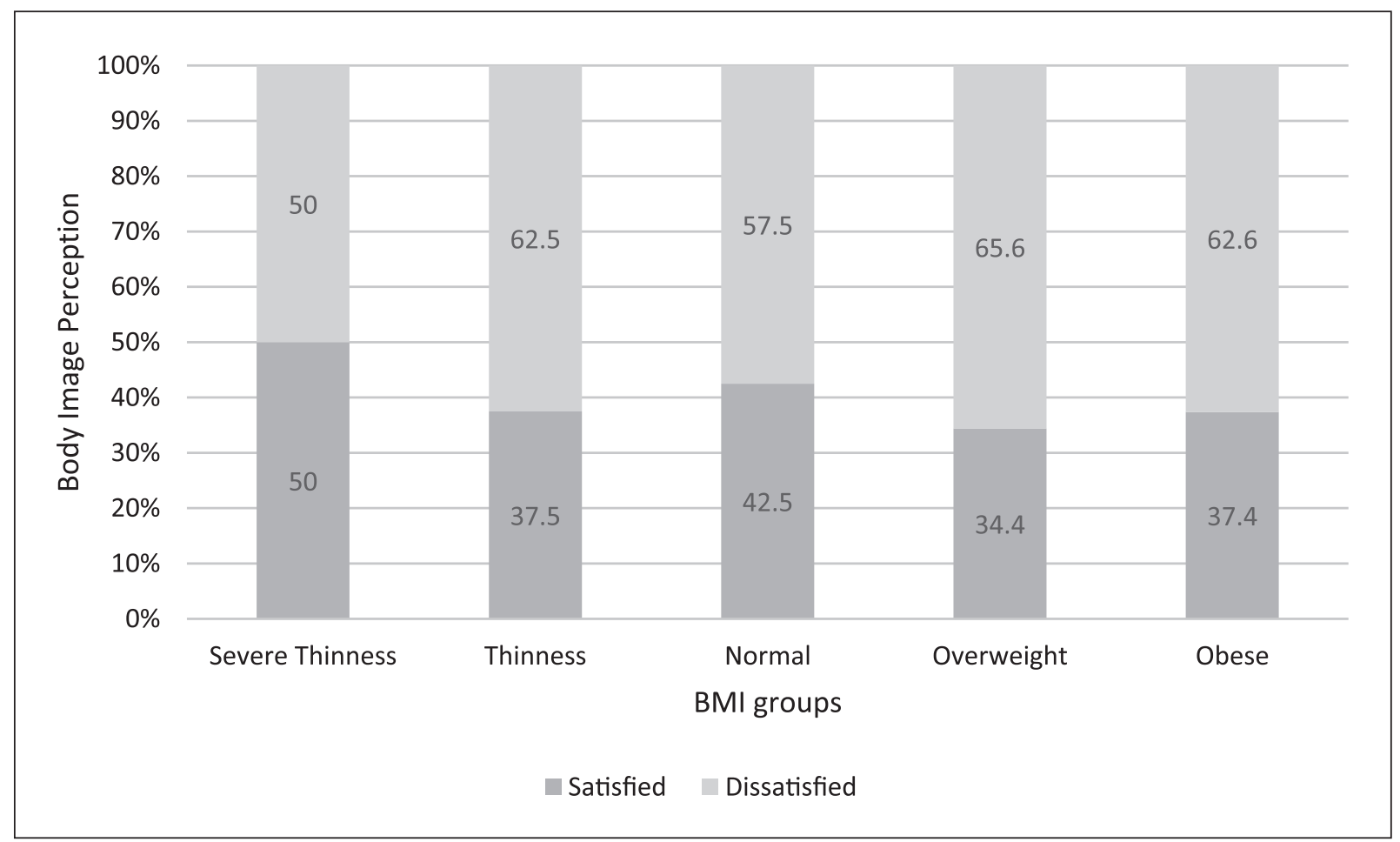

Fig. 2. Comparison between body image perception among different BMI groups of the participants (\%). 
Table 2. Association between weight status and body image perception among respondents ( $n$, \%)

\begin{tabular}{lcrr}
\hline Body image perception & Satisfied $n(\%)$ & Dissatisfied $n(\%)$ & $\mathrm{X}^{2}$ statistic (df) \\
\hline Weight status & & & \\
$\quad$ Normal BMI $(n=127)$ & $54(73.0)$ & $73(58.9)$ & $4.007(1)$ \\
$\quad$ Overweight / obese $(n=71)$ & $20(27.0)$ & $51(41.1)$ & 0.045 \\
\hline
\end{tabular}

Pearson's Chi-Square test was applied.

Table 3. Factors affecting BMI among participants

\begin{tabular}{|c|c|c|c|c|c|}
\hline $\begin{array}{l}\text { Dependent } \\
\text { variable }\end{array}$ & Independent variable & $\beta(95 \% \mathrm{Cl})$ & t-statistics & $p$-value & $\mathrm{R}^{2}$ \\
\hline \multirow[t]{10}{*}{ BMl } & Constant & $25.602(20.353,30.851)$ & 9.622 & 0.000 & 0.107 \\
\hline & Parental acceptance & $-0.096(-0.329,0.137)$ & -0.814 & 0.417 & \\
\hline & Peer acceptance & $0.230(-0.017,0.478)$ & 1.835 & 0.068 & \\
\hline & Importance of body shape & $0.059(-0.388,0.506)$ & 0.260 & 0.795 & \\
\hline & Comparison of body shape & $-0.570(-0.964,-0.175)$ & -2.851 & $0.005^{*}$ & \\
\hline & Body shape confidence & $0.292(-0.169,0.752)$ & 1.249 & 0.213 & \\
\hline & Body shape satisfaction & $0.014(-0.352,0.379)$ & 0.074 & 0.941 & \\
\hline & Body weight satisfaction & $-0.211(-0.540,0.117)$ & -1.269 & 0.206 & \\
\hline & Body weight and shape anxiety & $0.148(-0.210,0.506)$ & 0.814 & 0.417 & \\
\hline & Body weight and shape preoccupation & $-0.089(-0.369,0.204)$ & -0.568 & 0.570 & \\
\hline
\end{tabular}

A multiple Linear Regression test was applied.

* significant at $p<0.01$.

significant value $(p=0.005)$. Based on the Multiple Linear Regression statistical test, it can be concluded that there is a significant linear relationship between BMI and domain comparison of body shape $(p<0.01)$. It is observed that for every 1 unit increase in comparison of body shape score, there will be a 0.570 unit decrease in BMI value. Hence, the participant's BMI will be decreased by $0.570 \mathrm{~kg} / \mathrm{m}^{2}$ for every 1 unit increment in the domain of comparison of body shape score. Therefore domain comparison of body shape is the significant predicting factor for BMI $[\mathrm{BMI}=25.602-0.570$ (comparison of body shape) $]$.

\section{DISCUSSION}

According to NHMS 2019, the prevalence of overweight and obese children aged between 5 to 17 years old was $29.8 \%$ of which $15.0 \%$ were overweight while, $14.8 \%$ were obese (IPH, 2009). This is in contrast to the findings of the current study. The prevalence for overweight and obese children was $27.8 \%$ and $1.0 \%$, respectively. In a study conducted by Poh et al. (2016) the prevalence for obese adolescents is $12.6 \%$ which is higher than both the NHMS, $7.3 \%$, and the current study which is $7.6 \%$. But to note that, the NHMS 2011 (IPH, 2011) study and study by Poh et al. (2016) were conducted among both genders in different areas across the country meanwhile the current study was conducted only among female adolescents in Kuala Nerus district in Terengganu. Also to note that both the studies were conducted using BMI as the main indicator in calculating the prevalence of overweight and obesity among children and adolescents. The reason for only taking females as study participants are that a previous study that used the Attitudes towards body image questionnaire validated their questionnaire among female participants only.

As for all the age groups, all of the adolescents were in the normal category for body fat percentage. According to a study conducted in central and northern regions of peninsular Malaysia by $\mathrm{Ng}$ et al. (2019) the percent body fat in female adolescents is $29.4 \%$. Meanwhile, the present study reported that the mean body fat percent is $28.6 \%$ (7.3). Thus, it is lower than the previous findings of Ng's studies. A similar study conducted in Peninsular Malaysia in 2012 and 2014 found that the prevalence of percent body fat is $23.7 \%$ and $27.7 \%$, respectively (Majid et al., 2016). These data show that there is an increasing trend in percent body fat among female adolescents over a few years back. Interestingly, another study conducted by Noradilah et al. (2016) in Kuala Lumpur, Malaysia stated that the mean percent body fat for female adolescents is $33.4 \%$ (7.6). This is higher than the finding of the present study and MyHeART study findings. But this prevalence 
was calculated using the Dual Energy X-Ray Absorptiometry (DXA) method. The study concluded that the percent body fat measured using the BIA was underestimated than the DXA measurement. But there is no extreme bias between the DXA and BIA methods (Noradilah et al., 2016). Since the gold standard for analyzing body composition is the DXA method, there is a study conducted in Texas in the year 2017 stated that the BIA method underestimated fat mass relative to DXA in normal-weight people (Tinsley et al., 2017). The differences vary as the two technologies use different principles to operate, often the results are interchangeable. However, both DXA and BIA method is being widely used in studies subjected to availability and study budget.

A study among school children in the UK reported that $45 \%$ of the participants compared themselves with their peers in terms of body shape and size. The same study also stated that almost half of the children described as having negative body image perception if compared with their favorite celebrities (Tatangelo \& Ricciardelli, 2015). Due to emerging technologies, nowadays people are exposed to the thin-ideal body as what being portrait as beauty on various platforms on social media, especially on Facebook and Instagram. A study among students in Australia found a significant correlation between usage of Facebook and body image dissatisfaction $(r=0.57, p<0.001)$ (Fardouly \& Vartanian, 2014). Similarly, a study among $7^{\text {th }}$ Grade school children in the United States (US) reported that when adolescents are exposed to the thin ideal image, the consequence is that the occurrence of social comparison which led to body image dissatisfaction (Rodgers et al., 2015). The development of social comparison that affects the development of one's body image is crucial and critical in the adolescent life stage. As they grow older, this trait will have an effect on how they develop their self-esteem and confidence and also their eating behavior (Fardouly \& Vartanian, 2014). A healthy eating habit would be good, otherwise, it will indulge adolescents into an unhealthy eating habit that can lead to disordered eating behavior which can affect their body composition thus decreasing their potential growth due to inadequate nutrients to support their growth. Thus, this current study's finding has added new knowledge of comparison of body shape among the female adolescents can be predicting factors for the BMI of the adolescents.

What can be inferred from these findings is that, when an adolescent experience increasing age and growth, they increase their concern regarding their body image. They start to look and care for their body image. Generally, girls start to experience puberty at the age of 10 to 14 years old. So the physical changes will make them focus more on their body image and raise their concern for body image (Ricciardelli \& Yager, 2016). This finding is similar to other previous studies as reported indeed there is an association between age group and body image perception among female adolescents (Dion et al., 2015). Also, there is a significant association in body image perception based on their BMI $(p=0.045)$. Thus, it can be concluded that there is a significant association between weight status and body image perception among female adolescents. In another study conducted among Iranian women in Iran, the finding is consistent with the present study (Saeed et al., 2019). Similarly, a study by Shloim et al. (2013) also found that there is a relationship between BMI and body image among women. Then, another study, also reported significantly higher body image dissatisfaction was observed among the respondents of the study (Shloim et al., 2013). Besides, a study among school children also reported the same finding as to the current study where there is an association between BMI and body image dissatisfaction (Duchin et al., 2014). In addition, a study of African American high school students found a significant association between BMI classification and body image perception, with odds ranging from 3.40 to 13.60 (Allen et al., 2016). All the above-mentioned studies were conducted in different countries among different populations but still showed a significant association between BMI and body image perception, similar to the current study.

The limitation of this study is that this study was conducted in a single and short period. As the design for this study is a cross-sectional study. There was no follow-up study conducted with the participants. Next, the sample size for this study is only 198 respondents which are smaller when compared to other studies. Then, the respondents also only consist of female adolescents, so the comparison between gender cannot be made as such we cannot assess the body image perception among male adolescents. The distribution of race and ethnic group was also limited to only Malay ethnicity. Besides, the distribution of primary and secondary school adolescents also was not equally distributed due to financial and time constraints. The questionnaire used in this study was Attitudes towards Body Image adapted from MBSRQ and Stunkard's Figure Rating Scale. So, there may be selfreport bias and a tendency for the respondents to report an ideal answer. Despite the limitation of the current study, there is also a strength regarding this study. This study is the first study conducted in Kuala Nerus regarding body image perception and body fat percentage among adolescents. The finding from this study may be useful for further study to 
assess body image perception and its association with an eating disorder. Furthermore, communities in Kuala Nerus especially adolescents who participated in this health assessment study can be benefited and able to understand more about their BMI and body fat percentage and review their body image perception. Therefore, they can focus to improve on their overall psychological and mental health regarding body image perception and practice healthy eating habits.

\section{CONCLUSION}

In summary, the prevalence for overweight and obese adolescents is $27.8 \%$ and $1.0 \%$, respectively. There is a significant association between weight status noted as Body Mass Index (BMI) and body image perception $(p<0.045)$. The domain of comparison of body shape is found to be a predicting factor for BMI among female adolescents $(p<0.005)$. In conclusion, further study should be carried out to investigate the factors that can lead to the development of dissatisfaction with body image and the effects of getting negative body image in life of the adolescents during their adolescent's phase.

\section{ACKNOWLEDGEMENTS}

This project was partially funded by the UniSZA DPU Research Grant Scheme (UniSZA/2017/DPU/43). The authors would like to thank all students who willingly participated in this study and gave great cooperation during this study. We are also grateful to the school principals, all teachers, and the Ministry of Education for giving us permission and cooperation to conduct this study.

\section{REFERENCES}

Allen, D., Belcher, H.M.E. \& Young, A. 2014. BMI, Body image, emotional wellbeing and weight control behaviors in urban African-American adolescents. International Journal of Child Health Nutrition, 5(3): 55-104.

Brown, J.E. 2019. Nutrition Throughout the Life Cycle. Cengage Learning. United States of America. 319 pp.

Crishel, D.K. 2006. The relationship between body image and body composition among male and female college students. (Ph.D). Oklahoma State University.
David, P. 1994. Invited essay; What is body image? Behaviour Research Therapy, 32(5): 497-502.

Dion, J., Blackburn, M.E. \& Auclair, J. 2015. Development and aetiology of body dissatisfaction in adolescents boys and girls. International Journal of Adolescents and Youth, 20(2): 151-166.

Duchin, O., Marin, C. \& Mora-Plazas, M. 2014. A prospective study of body image dissatisfaction and BMI change in school age children. Public Health Nutrition, 18(2): 322-8.

Fardouly, J. \& Vartanian, L.R. 2014. Negative comparisons about one's appearance mediate the relationship between Facebook usage and body image concern. Body Image, 12:82-88.

Guinn, B., Semper, T. \& Jorgensen, L. 1997. Body image perception in female Mexican-American adolescents. Journal of School Health, 67(3): 112-117.

Institute for Public Health (IPH) National Institutes of Health, Ministry of Health Malaysia (MOH). 2011. National Health Morbidity Survey (NHMS) [WWW Document] URL http://www.iku.gov.my/ nhms-2011 (accessed 12.10.19)

Institute for Public Health (IPH) National Institutes of Health, Ministry of Health Malaysia (MOH). 2019. National Health Morbidity Survey (NHMS) [WWW Document] URL http://www.iku.gov.my/ nhms-2019 (accessed 1.16.20)

Khor, G.L. 2009. Perceptions of body image among male and female adolescents. Singapore Medical Journal, 50(3): 303-314.

Majid, H.A., Amiri, M. \& Azmi, N.M. 2016. Physical activity, body composition and lipid changes in adolescents: Analysis from MyHeART study. Nature Scientific Report, 6: 1-8.

Ng, A.K., Hairi, N.N. \& Jalalludin, M.Y. 2019. Dietary intake, physical activity and muscle strength among adolescents: The Malaysian Health and Adolescents Longitudinal Research Team (MyHeART) Study. BMJ Open, 9(6): 1-8.

Noradila, M.J., Ang, Y.N. \& Kamarudin, N.A. 2016. Assessing body fat of children by skinfold thickness, bio-electrical impedance analysis, Dual-energy X-ray Absorptiometry: A validation study among malay children aged 7 to 11 years old. Asia Pacific Journal of Health, 28(5): 7484.

Poh, B.K., Weng, J.E. \& Abdul, A.K. 2016. Difference in body build in children of different ethnic group and their impact on the prevalence of stunting, thinness, overweight and obesity. Food and Nutrition Bulletin, 37(1): 3-13. 
Reel, J., Voelker, D. \& Breenleaf, C. 2015. Weight status and body image perception in adolescents. Adolescents Health, Medicine and Therapeutics, 25(6): 149-158.

Ricciardelli, L.A. \& Yager, Z. 2016. Adolescence body image: From development to preventing dissatisfaction. Advances in eating disorder. New York. 229-230 pp. (English).

Rodgers, R.F., Mcclean, S.A. \& Paxton, S.J. 2015. Longitudinal relationships among internalization of the media ideal, peer social comparison and body dissatisfaction: Implications for the tripartite influence model. Developmental Psychology, 51(5): 706-719.

Saeed, P.S., Sadat, A.A. \& Sim, O.F. 2019. The effect of religiosity on the relationship between BMI and body image among Iranian women. Psychology of Religion and Spirituality, 11(2): 168-175.

Shloim, N., Hetherington, M.M. \& Rudolf, M. 2013. Relationship between body mass index and women's body image, self-esteem and eating behaviors in pregnancy: A cross sectional study. Jounal of Health Psychology, 20(4): 413426.

Tatangelo, G.L. \& Ricciardelli, L.A. 2015. Children's body image and social comparisons with peers and the media. Journal of Health Psychology, 22(6): 776-787.

Taylor, R.W., Jones, I.E., William, S.M. \& Goulding, A. 2002. Body fat measured by Dual-Energy $\mathrm{X}$-ray Absorptiometry corresponding to recently recommended Body Mass Index (BMI) cutoffs for overweight and obesity in children and adolescents aged 3-18 years. The American Journal of Nutrition, 76(6): 1416-1421.
Tinsley, G.M., Forsse, J.F., Morales, E. \& Grandjean, P. 2017. Bias between DXA and BIA varies based on quantity of fat mass and fat-free mass. Medicine and Science in Sports and Exercise, 49(1): 485-489.

Tylka, T.L. \& Wood-Barcalow, N.L. 2015. What is and what is not body image? Conceptual foundations and construct definition. Body Image, 14: 118-129.

Vannesa, V.E. 2014. Beauty Standards: See how body types changes through history. [WWW Document] URL https://www.scienceofpeople. com/beauty-standards/ (assessed 9.24.19)

Wang, Y. \& Chen, X. 2012. Is ideal body image related to obesity and lifestyle behaviors in African-Mexican adolescents? Child Care Health Development, 38(2): 219-228.

World Health Organization (WHO). 2017. Growth Reference BMI for age (5-19 years). [WWW Document] URL https://www.who.int/tools/ growth-reference-data-for-5 to 19-years/ indicators/bmi-for-age (assessed 9.10.19)

World Health Organization (WHO). 2021. Adolescents and Young Adults Health Keyfact. WHO Factsheet. [WWW Document] URL https://www.who.int/news-room/fact-sheets/ detail/adolescents-health-risks-and-solutions (assessed 6.15.21) 